\title{
INFLUÊNCIA DO CORPO LÚTEO SOBRE A RECUPERAÇÃO DE OÓCITOS IMATUROS BOVINOS DERIVADOS DE FÊMEAS POST-MORTEM
}

\author{
M. V. O. SANTOS, L. B. QUEIROZ NETA, A. A. BORGES, M. B. SILVA e A. F. PEREIRA*
$\begin{gathered}\text { Universidade Federal Rural do Semi-Árido } \\ \text { alexsandra.pereira@ufersa.edu.br }{ }^{*}\end{gathered}$ \\ Submetido 15/01/2017 - Aceito 02/10/2017 \\ DOI: $10.15628 /$ holos.2017.5556
}

\section{RESUMO}

O objetivo do presente trabalho foi avaliar a influência do corpo lúteo $(\mathrm{CL})$, glândula endócrina temporária presente nos ovários, sobre a recuperação de oócitos imaturos bovinos derivados de ovários de fêmeas postmortem. Para tanto, as seguintes comparações foram realizadas: fêmeas não prenhes com $\mathrm{CL}$ vs. fêmeas não prenhes sem $\mathrm{CL}$; fêmeas prenhes com $\mathrm{CL}$ vs. fêmeas não prenhes com $\mathrm{CL}$; e ovário com $\mathrm{CL}$ vs. ovário sem $\mathrm{CL}$. Assim, ovários foram obtidos em abatedouro e os oócitos recuperados por aspiração folicular. A qualidade oocitária foi avaliada por morfologia convencional e ensaio de azul cresil brilhante (ACB), sendo quantificados oócitos viáveis e não viáveis para cada técnica. Todos os dados foram analisados pelo teste de Fisher $(P<0,05)$. Assim, avaliando fêmeas ou ovários com ou sem $C L$, nenhuma diferença foi observada nos parâmetros avaliados. Contudo, um maior percentual de oócitos viáveis por morfologia foi obtido de fêmeas prenhes com $\mathrm{CL}$, quando comparado às fêmeas não prenhes com $\mathrm{CL}$. Em conclusão, pode-se afirmar que parâmetros de recuperação e qualidade oocitária não são afetados pela presença de $\mathrm{CL}$, exceto em doadoras prenhes que apresentaram oócitos de melhor qualidade.

PALAVRAS-CHAVE: azul de cresil brilhante, competência oocitária, morfologia convencional.

\section{INFLUENCE OF THE CORPUS LUTEUM ON THE RECOVERY OF BOVINE IMMATURE OOCYTES DERIVED FROM POST-MORTEM FEMALES}

\begin{abstract}
The aim of the present study was to evaluate the influence of the corpus luteum $(\mathrm{CL})$, temporary endocrine gland present on the ovaries, on the recovery of bovine immature oocytes derived from ovaries of post-mortem females. Thun, the following comparisons were performed: non-pregnant females with $\mathrm{CL}$ vs. females not-pregnant without $\mathrm{CL}$; pregnant females with $\mathrm{CL}$ vs. not-pregnant females with $\mathrm{CL}$; and ovary with $\mathrm{CL}$ vs. ovary without $\mathrm{CL}$. So, ovaries were obtained in slaughterhouse and the oocytes recovered by follicular aspiration. The oocyte quality was evaluated by conventional morphology and brilliant cresyl blue assay
\end{abstract}

$(\mathrm{BCB})$, and viable and non-viable oocytes were quantified for each technique. All data were analyzed by Fisher test $(P<0.05)$. Thus, evaluating females or ovaries with or without $\mathrm{CL}$, no difference was observed in the evaluated parameters. Nevertheless, a higher percentage of viable oocytes by morphology were obtained from pregnant females with $\mathrm{CL}$ comparing nonpregnant females with $\mathrm{CL}$. In conclusion, it can be stated that it is possible that recovery parameters and oocyte quality are not affected by the presence of $\mathrm{CL}$, except in pregnant donors who presented oocytes of better quality.

KEYWORDS: brilliant cresyl blue, conventional morphology, oocyte competence. 


\section{INTRODUÇÃO}

As biotécnicas reprodutivas têm despertado grande interesse dos setores científicos e comerciais em virtude dos inúmeros benefícios que podem promover para o melhoramento genético e aumento da produtividade de rebanhos (Azevedo, Magalhães, Oliveira, Lima, \& Magalhães, 2015). Dentre essas biotécnicas, a produção in vitro de embriões (PIVE) merece destaque, pois possibilita a obtenção de um grande número de embriões viáveis para a produção animal e investigação científica (Nivet et al., 2012; Carrocera, Caamaño, Trigal, Martín, \& Díez, 2016).

Em geral, a PIVE é composta por algumas etapas, iniciando com a colheita e seleção de oócitos imaturos, maturação in vitro (MIV) desses oócitos, fecundação in vitro (FIV) com espermatozoides, desenvolvimento in vitro (DIV) dos embriões e transferência para receptoras sincronizadas (Paramio \& Izquierdo, 2016). Assim, visando aperfeiçoar essas etapas e aumentar ainda mais o sucesso da PIVE, vários estudos têm sido realizados avaliando fatores relacionados desde os momentos iniciais de colheita oocitária, a fim de obter oócitos em quantidade e qualidade adequados (Barbosa, Toniollo, \& Guimarães, 2013), até as etapas finais de desenvolvimento embrionário (Zullo et al., 2016). Nesse contexto, alguns fatores podem influenciar a qualidade e competência de oócitos imaturos, incluindo características intrínsecas da fêmea, normalmente relacionadas a parâmetros fisiológicos ovarianos, como a presença do corpo lúteo e estádio do ciclo estral (Hajarian, Shahsavari, Karami-Shabankareh, \& Dashtizad, 2016).

O corpo lúteo $(\mathrm{CL})$ pode ser considerado um indicador do estádio reprodutivo da fêmea, uma vez que essa estrutura endócrina é formada após a ovulação e está envolvida na manutenção da gestação e regulação do ciclo estral (Penitente-Filho et al., 2014). A presença ou ausência do CL na superfície ovariana têm se mostrado um critério de seleção macroscópico para recuperação de oócitos em bovinos (Pirestani et al., 2011; Hajarian et al., 2016), bubalinos (El-Naby et al., 2013) e ovinos (Davachi, Kohram, \& Zeinoaldini, 2013). Contudo, os resultados com relação à influência do CL sobre a qualidade oocitária ainda são controversos (Penitente-Filho et al., 2015).

Logo, o objetivo do presente trabalho foi avaliar a influência do CL na superfície ovariana sobre a recuperação de oócitos imaturos bovinos derivados de ovários de fêmeas post-mortem.

\section{MATERIAL E MÉTODOS}

Esse estudo foi aprovado pelo Comitê de Ética de Uso de Animais da Universidade Federal Rural do Semiárido (CEUA/UFERSA; no do processo: 23091.001069/2015-79). Os experimentos foram conduzidos no Laboratório de Biotecnologia Animal (LBA/UFERSA).

O trabalho foi dividido em três comparações, avaliando a influência do corpo lúteo (CL) nos ovários de fêmeas post-mortem sobre os parâmetros de recuperação oocitária: (i) fêmeas não prenhes com $C L$ visível vs. fêmeas não prenhes sem $C L$ visível; (ii) fêmeas prenhes com $C L$ visível vs. fêmeas não prenhes com $\mathrm{CL}$ visível e (iii) ovário com $\mathrm{CL}$ vs. ovário sem $\mathrm{CL}$. Para todas as comparações, a qualidade oocitária foi determinada por avaliação morfológica convencional e ensaio de azul cresil brilhante (ACB). 
Assim, para atender o delineamento proposto anterior, ovários de fêmeas bovinas foram coletados em abatedouro local (Mossoró, Rio Grande do Norte) e os mesmos foram avaliados quanto à presença de $\mathrm{CL}$ visível na superfície ovariana e armazenados separadamente, de acordo com os grupos experimentais. Além disso, fêmeas gravídicas foram identificadas após o abate por exame visual de membranas fetais, vesículas amnióticas, cotilédones e presença de feto. Os ovários obtidos foram transportadas ao laboratório em solução salina ( $\mathrm{NaCl}, 0,9 \%$ ) acrescido de antibióticos a uma temperatura de $35-37^{\circ} \mathrm{C}$.

No laboratório, os ovários foram lavados com uma nova solução de transporte e os folículos visíveis com diâmetro de 2-8 $\mathrm{mm}$ foram aspirados utilizando uma agulha $21 \mathrm{G}$ acoplada a uma seringa de 5,0 mL. Após a aspiração folicular, os oócitos foram classificados sob estereomicroscópio por critérios morfológicos em quatro graus: grau I (três ou mais camadas de células do cumulus e citoplasma homogêneo), grau II (uma ou duas camadas de células do cumulus e citoplasma homogêneo), grau III (camada incompleta de células do cumulus e citoplasma homogêneo ou heterogêneo) e grau IV (células do cumulus com aspecto gelatinoso ou oócito degenerado com grânulos escuros) (Gonçalves et al., 2008). Os oócitos classificados como graus I e II foram considerados viáveis e graus III e IV classificados como não viáveis.

Os oócitos selecionados por critérios morfológicos foram lavados em gotas de solução tampão fosfato, PBS (200 $\mu \mathrm{L}$ ) e incubados em gotas de $300 \mu \mathrm{L}$ de ACB diluído em PBS ( $26 \mu \mathrm{M}$, Sigma, EUA) por $60 \mathrm{~min}$ a $38,5^{\circ} \mathrm{C}$. Decorrido esse tempo, os oócitos foram lavados novamente em gotas de PBS $(200 \mu \mathrm{L})$ e visualizados sob estereomicroscópio. O corante ACB se baseia na atividade da enzima glucose-6-fosfato desidrogenase (G6PDH) que se encontra mais ativa em oócitos em fase de crescimento, reduzindo o corante de azul para incolor $\left(A C B^{-}\right)$. Em oócitos crescidos, a atividade da G6PDH diminui e o $A C B$ não é reduzido fazendo com que os oócitos apresentem cor azul no citoplasma ( $\mathrm{ACB}^{+}$) (Bhojwani, Alm, Torner, Kanitz, \& Poehland, 2007). Assim, oócitos com coloração azul em seu citoplasma foram classificados como $\mathrm{ACB}^{+}$e considerados competentes. Já àqueles com citoplasma incolor foram classificados como $\mathrm{ACB}^{-}$e considerados menos competentes (Opiela \& Katska-Ksiażkiewicz, 2013).

Para a análise estatística, a taxa de recuperação oocitária (relação entre o número de oócitos recuperados e o número de folículos aspirados), o número de oócitos colhidos por ovário e o percentual de oócitos viáveis (número de oócitos viáveis sobre o total de oócitos recuperados) por morfologia e ensaio de ACB foram avaliados pelo teste exato de Fischer $(P<0,05)$, usando o software Graphpad Instat 3.06 (GraphPad Software Inc., La Jolla, EUA). Um total de quinze repetições foi realizado, sendo cinco repetições por cada comparação.

\section{RESULTADOS E DISCUSSÃO}

Na comparação entre fêmeas não prenhes com $C L$ visível e as fêmeas não prenhes sem $\mathrm{CL}$ visível, a taxa de recuperação e o número de oócitos por ovário foram semelhantes $(P>0,05)$, bem como o percentual de oócitos viáveis por morfologia convencional e ensaio de ACB (Tabela 1).

De forma similar, Pirestani et al. (2011) não observaram diferenças no percentual de oócitos recuperados de doadoras com $\mathrm{CL}$ ou sem $\mathrm{CL}$, independentemente da presença de folículo 
dominante. Em contrapartida, Reis, Metelo, Santos e Silva (2006). (2006) demonstraram que a taxa de recuperação e qualidade de oócitos bovinos foi maior em fêmeas em fase luteínica do que em fase folicular.

Tabela 1: Recuperação e qualidade de oócitos bovinos derivados de fêmeas não prenhes com ou sem CL visível.

\begin{tabular}{ccccc}
\hline $\begin{array}{c}\text { Presença } \\
\text { de CL }\end{array}$ & $\begin{array}{c}\text { Taxa de recuperação } \\
\text { (\%) }\end{array}$ & $\begin{array}{c}\text { Oócitos/ } \\
\text { ovário }\end{array}$ & $\begin{array}{c}\text { Oócitos viáveis por } \\
\text { morfologia (\%) }\end{array}$ & $\begin{array}{c}\text { Oócitos viáveis pelo } \\
\text { ensaio de ACB (\%) }\end{array}$ \\
\hline+ & $325 / 487$ & 9,0 & 208 & 176 \\
& $(66,73)$ & & $(64,00)$ & $(54,15)$ \\
- & $390 / 620$ & 7,5 & 241 & 221 \\
$(62,90)$ & $(61,79)$ & $(56,66)$ \\
\hline
\end{tabular}

$\mathrm{P}>0,05$.

Quando oócitos de fêmeas prenhes com $\mathrm{CL}$ e fêmeas não prenhes com CL foram comparados, não houve diferença nos parâmetros de recuperação $(P>0,05)$. Contudo, na avaliação morfológica foi obtido um maior $(\mathrm{P}<0,05)$ percentual de oócitos viáveis de fêmeas prenhes com $\mathrm{CL}$ do que de fêmeas não prenhes com CL (Tabela 2).

Tabela 2: Recuperação e qualidade de oócitos bovinos oriundos de fêmeas com CL prenhes e não prenhes.

\begin{tabular}{cccccc}
\hline $\begin{array}{c}\text { Animal } \\
\text { prenhe }\end{array}$ & $\begin{array}{c}\text { Presença } \\
\text { de CL }\end{array}$ & $\begin{array}{c}\text { Taxa de } \\
\text { recuperação (\%) }\end{array}$ & $\begin{array}{c}\text { Oócitos } \\
\text { /ovário }\end{array}$ & $\begin{array}{c}\text { Oócitos viáveis por } \\
\text { morfologia (\%) }\end{array}$ & $\begin{array}{c}\text { Oócitos viáveis pelo } \\
\text { ensaio de ACB (\%) }\end{array}$ \\
\hline+ & + & $\begin{array}{c}465 / 782 \\
(59,46)^{\mathrm{a}}\end{array}$ & $8,2^{\mathrm{a}}$ & $\begin{array}{c}293 \\
(63,01)^{\mathrm{a}}\end{array}$ & $\begin{array}{c}\text { a } \\
87\end{array}$ \\
& & $\begin{array}{c}165 / 256 \\
(64,45)^{\mathrm{a}}\end{array}$ & $4,9^{\mathrm{a}}$ & $\begin{array}{c}87 \\
(52,72)^{\mathrm{b}}\end{array}$ & $\begin{array}{c}\mathrm{b} \\
(44,24)^{\mathrm{a}}\end{array}$ \\
\hline
\end{tabular}

a,b: diferem na coluna $(P<0,05)$.

Estes números concordam em parte com os resultados obtidos por Barbosa et al. (2013) que não observaram diferença na média de oócitos recuperados $(31,34$ vs. 44,08) e viáveis por morfologia $(38,51$ vs. 32,00). Takuma et al. (2010) também não observaram diferença na viabilidade e percentual de oócitos obtidos de doadoras gravídicas ou não gravídicas; contudo, um maior percentual de embriões clivados $(74,3$ vs. 63,3$)$ e blastocistos $(46,9$ vs. 33,4$)$ foi obtido de fêmeas em estado gestacional, mostrando que o estádio fisiológico pode influenciar a competência de desenvolvimento de oócitos.

Finalmente, ovários com e sem $\mathrm{CL}$ do mesmo animal não apresentaram diferenças com relação a parâmetros de recuperação e qualidade oocitária por morfologia convencional e ensaio de ACB ( $P>0,05)$ (Tabela 3), estando de acordo com os resultados de Barbosa et al. (2013).

Sugulle, Dochi e Koyama (2008) também avaliaram a qualidade dos oócitos de ovários com e sem $C L$, utilizado o ensaio de $A C B$, e, assim como neste trabalho, não foi encontrada diferença no percentual de oócitos $\mathrm{ACB}^{+}(73,9$ vs. 69,5). Em contraste, Hajarian et al. (2016) obtiveram um maior percentual de oócitos $\mathrm{ACB}^{+}$a partir de ovários sem $\mathrm{CL}$, comparado a ovários com $\mathrm{CL}$ (57,3 vs. 44,1), bem como uma taxa de desenvolvimento embrionário superior. Em soma, Sugulle et al. (2008) ressaltaram que a classificação com o ensaio de ACB pode facilitar a seleção de oócitos 
competentes para a fecundação in vitro, auxiliando o método de seleção morfológica convencional.

Tabela 3: Recuperação e qualidade de oócitos bovinos derivados de ovários com ou sem CL.

\begin{tabular}{ccccc}
\hline $\begin{array}{c}\text { Superfície } \\
\text { ovariana }\end{array}$ & $\begin{array}{c}\text { Taxa de recuperação } \\
(\%)\end{array}$ & $\begin{array}{c}\text { Oócitos/ } \\
\text { ovário }\end{array}$ & $\begin{array}{c}\text { Oócitos viáveis por } \\
\text { morfologia (\%) }\end{array}$ & $\begin{array}{c}\text { Oócitos viáveis pelo } \\
\text { ensaio de ACB (\%) }\end{array}$ \\
\hline Com CL & $265 / 436$ & 8,8 & 187 & 137 \\
& $(60,77)$ & & $(70,56)$ & $(51,69)$ \\
Sem CL & $280 / 445$ & 10,0 & 197 & 143 \\
& $(62,92)$ & & $(70,35)$ & $(51,07)$ \\
\hline
\end{tabular}

$\mathrm{P}>0,05$.

\section{CONCLUSÕES}

A taxa de recuperação, o número de oócitos por ovário e a qualidade oocitária por critérios morfológicos e ensaio de $A C B$ não foram afetados pela presença do $C L$, exceto em caso de doadoras gravídicas, aumentando a qualidade dos oócitos recuperados.

\section{AGRADECIMENTOS}

Este estudo foi apoiado pelo Conselho Nacional de Desenvolvimento Tecnológico e Científico (CNPq, processo no. 477710/2013-1).

\section{REFERÊNCIAS}

Azevedo, T. I. K., Magalhães, L. F., Oliveira, F. C., Lima, G. P., \& Magalhães, G. M. (2015). A relação da evolução das técnicas de criação e o advento das biotecnologias da reprodução com a incidência de distocias em bovinos. Investigação, 14(1), 91-97.

Barbosa, C. P., Toniollo, G. H., \& Guimarães, E. C. (2013). Produção in vitro de embriões de bovinos da raça Nelore oriundos de ovócitos de ovários com e sem corpo lúteo. Ciência Animal Brasileira, 14(1), 81-90.

Bhojwani, S., Alm, H., Torner, H., Kanitz, W., \& Poehland, R. (2007). Selection of developmentally competent oocytes through brilliant cresyl blue stain enhances blastocyst development rate after bovine nuclear transfer. Theriogenology, 67(2), 341-345.

Carrocera, S., Caamaño, J. N., Trigal, B., Martín, D., \& Díez, C. (2016). Developmental kinetics of in vitro-produced bovine embryos: an aid for making decisions. Theriogenology, 85(5), 822-827.

Davachi, N. D., Kohram, H., \& Zeinoaldini, S. (2013). Effect of the presence of corpus luteum on the ovary and the new oocyte recovery method on the oocyte recovery rate and meiotic competence of ovine oocytes. African Journal of Biotechnology, 10(47), 9706-9709.

El-Naby, A. S. A. H., Mahmoud, K. G., Ahmed, Y. F., Abouel-Roos, M. E., \& Abdel-Ghaffar, A. E. (2013). Effect of season of the year and ovarian structures on oocytes recovery rate, quality and meiotic competence in egyptian buffaloes. Global Veterinária, 10(4), 408-412. 
Gonçalves, P. B. D., Oliveira, M. A. L., Mezzalira, A., Montagner, M. M., Visintin, J. A., \& Costa, L. F. S. (2008). Produção in vitro de embriões. In P. B. D. Gonçalves, J. R. Figueiredo, \& V. J. F. Freitas (Ed). Biotécnicas Aplicadas à Reprodução Animal (Vol. 2, Chap. 14, pp. 261-291). São Paulo: Roca.

Hajarian, H., Shahsavari, M. H., Karami-Shabankareh, H., \& Dashtizad, M. (2016). The presence of corpus luteum may have a negative impact on in vitro developmental competency of bovine oocytes. Reproductive Biology, 16(1), 47-52.

Nivet, A. L., Bunel, A., Labrecque, R., Belanger, J., Vigneault, C., Blondin, P., \& Sirard, M. A. (2012). FSH withdrawal improves developmental competence of oocytes in the bovine model. Reproduction, 143(2), 165-171.

Opiela, J., \& Katska-Ksiażkiewicz, L. (2013). The utility of brilliant cresyl blue (BCB) staining of mammalian oocytes used for in vitro embryo production (IVP). Reproductive Biology, 13(3), 177-183.

Paramio, M. T., \& Izquierdo, D. (2016). Recent advances in in vitro embryo production in small ruminants. Theriogenology, 86(1), 152-159.

Penitente-Filho, J. M., Carrascal, E., Oliveira, F. A., Zolini, A. M., Oliveira, C. T., Soares, Í. A. C., \& Torres, C. A. A. (2014). Influence of dominant follicle and corpus luteum on recovery of good quality oocytes for in vitro embryo production in cattle. British Biotechnology Journal, 4(12), 1305-1312.

Penitente-Filho, J. M., Jimenez, C. R., Zolini, A. M., Carrascal, E., Azevedo, J. L., Silveira, C. O., \& Torres, C. A. A. (2015). Influence of corpus luteum and ovarian volume on the number and quality of bovine oocytes. Animal Science Journal, 86(2), 148-152.

Pirestani, A., Hosseini, S. M., Hajian, M., Forouzanfar, M., Moulavi, F., \& Abedi, P. (2011). Effect of ovarian cyclic status on in vitro embryo production in cattle. International Journal of Fertility \& Sterility, 4(4), 172-175.

Reis, A., Metelo, R., Santos, P., \& Silva, F. M. (2006). Efeito da estrutura ovárica e da idade de bovinos da raça Holstein Friesian na quantidade e qualidade de ovócitos e de embriões produzidos in vitro. Brazilian Journal of Veterinary Research and Animal Science, 43(5), 629636.

Sugulle, A. H., Dochi, O., \& Koyama, H. (2008). Developmental competence of bovine oocytes selected by brilliant cresyl blue staining: effect of the presence of corpus luteum on embryo development. Journal of Mammalian Ova Research, 25(1), p. 50-55.

Takuma, T., Sakai, S., Ezoe, D., Ichimaru, H., Jinnouchi, T., \& Kaedei, Y. (2010). Effects of season and reproductive phase on the quality, quantity and developmental competence of oocytes aspirated from Japanese black cows. Journal of Reproduction and Development, 56(1), 55-59.

Zullo, G., Canditiis, C., Pero, M. E., Albero, G., Salzano, A., Neglia, G., \& Gasparrini, B. (2016). Crocetin improves the quality of in vitro-produced bovine embryos: Implications for blastocyst development, cryotolerance, and apoptosis. Theriogenology, 86(8), 1879-1885. 